\title{
Dynamic Optimization of Airflow Organization in Green Data Center Based on MATLAB
}

\author{
ZHAO Jinhuil $^{1}$, GUO Binjie ${ }^{2}$, QIAO Hongwei ${ }^{*}$ and ZHU Qingbo $^{2}$ \\ ${ }^{1}$ School of Mechanical and Power Engineering, Zhengzhou University, Zhengzhou, Henan Province, 450001, China \\ ${ }^{2}$ School of Chemical Engineering, Zhengzhou University, Zhengzhou, Henan Province, 450001, China
}

\begin{abstract}
In order to make data centers meet the requirements of green data center standards, this paper starts with air conditioning systems with high energy consumption, and dynamically optimizes airflow organization. It uses limited space convection heat transfer and air kinematics to dissipate multiple processes in the data center. The process is solved by MATLAB optimization and reverse demonstration. In this paper, the influence of cold air flow on cabinet cooling is divided into four key processes: mixing loss during descent, convective heat transfer in descending process, the convection heat exchange during the steady flow process, and mixed airflow discharge. By analyzing the temperature change of cold airflow in each process, and seeking the relationship between velocity $v$ and air resistance $F$ in viscous gas flow, the temperature-velocity relationship is obtained. Then the relationship between cold loss, PUE value and indoor height of the data center was analyzed to obtain the ceiling height corresponding to the standard cabinet height. The best combination is the ceiling height of $3.5 \mathrm{~m}$ and the cabinet height of $2 \mathrm{~m}$ and the corresponding PUE value is about 1.87, which meets the requirements of green data centers and is highly economical.
\end{abstract}

\section{Introduction}

With the continuous deepening and development of network and informatization construction, the number of various IT equipment continues to increase. In 2017 alone, about 8 million data centers around the world consumed 416.2 billion $\mathrm{kWh}$ of electricity, which is equivalent to the global total $2 \%$ of electricity consumption is expected to reach $3-4 \%$ of global electricity consumption by the end of 2020 . Specific to China's data centers, the total number has exceeded 400,000 , and the annual power consumption exceeds $1.5 \%$ of the entire society's power consumption. Most of these data centers have a PUE (Power Usage Effectiveness) value greater than 2.21 , which is in line with international advanced levels. Compared with 1.21, there is a large gap, and the energy-saving potential is huge. Therefore, China has proposed the construction of a green data center that can maximize energy efficiency and minimize environmental impact under the conditions of ensuring the safe, stable and reliable operation of the information system and its supporting equipment during the entire life cycle.

The energy consumption of data centers is mainly reflected in server equipment and refrigeration equipment, and the energy consumption of the refrigeration system accounts for about $30-50 \%$ of the total energy consumption of the entire data center operation. To optimize the energy consumption of data center refrigeration systems, promote the construction of green data centers. This article starts with improving the airflow organization, and by optimizing the installation height of the air-conditioning air outlets in the data center and improving the efficiency of the refrigeration system in the computer room, thereby reducing the energy consumption of refrigeration equipment, to serve the industry's green data center construction.

\section{PUE value and energy factor analysis}

According to the "Data Center Research"2, in this article we divide the PUE value into IT equipment energy consumption (ITEP), refrigeration equipment energy consumption $(F E P)$ and power supply energy consumption $(E L E P)$. This article defines PUE as:

$$
P U E=1+\frac{0.4 F E P+1.2 E L E P}{I T E P}
$$

In calculating PUE, the weighting factor of the energy consumption of IT equipment is 1.0, the weighting factor of cooling energy consumption is 0.4 , and the weighting factor of energy consumption of other equipment is 1.22 .

Assuming a certain amount of energy consumption in IT equipment and power supply, the most effective way to reduce PUE is to reduce cooling energy consumption. In order to reduce excess energy consumption and realize the construction of green data centers, this article adjusts the heat dissipation efficiency and changes the value of FEP, so as to achieve the purpose of reducing PUE.

\footnotetext{
*Corresponding author's e-mai: qhwpudding@163.com
} 


\section{Data center thermal analysis}

\subsection{Geometric Space in the Data Center}

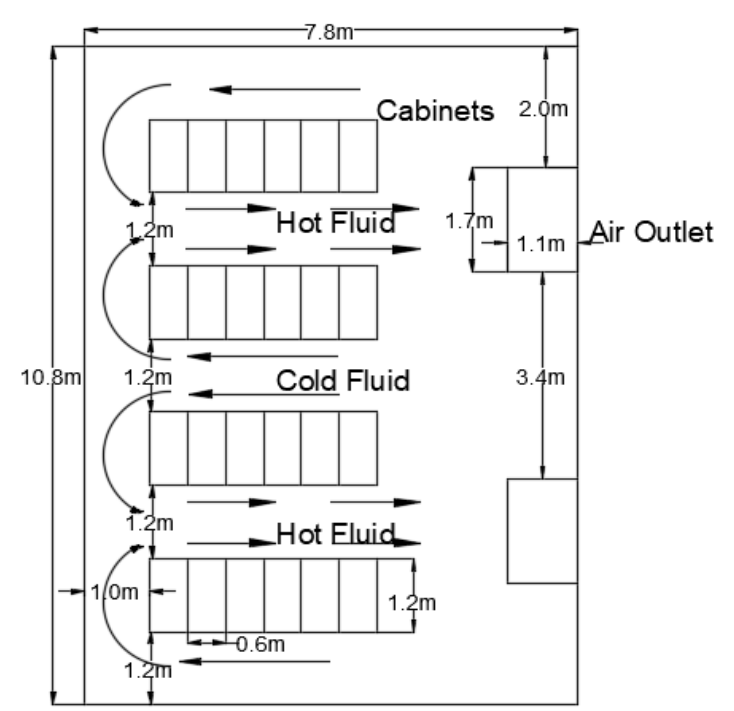

Figure 1. Schematic plan of the data center

From this plan view, the airflow in the data center can be obtained: the cabinets are arranged in four rows, and the hot and cold fluids flow separately. The cold air flows through four processes from the cooling port to the upper part of the cabinet, from the upper part of the cabinet to the ground, through the hot air flow channel, and as the hot air diffuses and discharges 3 .

This article will analyze the heat transfer and dynamics of these four processes, establish the airflow temperature model and speed model, and make a temperature-speed coupling relationship to find the effective heat exchange amount $Q$ of the cold airflow, and then the optimal solution of effective heat dissipation efficiency and PUE value is found.

The length of the cabinet in this paper is $600 \mathrm{~mm}$, the width of the cabinet is $1200 \mathrm{~mm}$, and the typical height 4 of the cabinet is 2.2 meters, 2.0 meters, 1.8 meters, 1.6 meters, 1.4 meters, 1.2 meters, and 1 meter.

\subsection{Temperature model of cold airflow}

First, the cold airflow is blown out from the cold air outlet at an initial temperature of $t_{0}$ and an initial velocity of $v_{0}=3.6 \mathrm{~m} / \mathrm{s}$, and performs convective heat exchange with the hot airflow of temperature $t_{m}$ at the top of the cabinet, the temperature rises to $t_{1}$, and is also affected by air resistance. The speed is reduced,thus, it can be obtained that:

$$
t_{1}=t_{0}+\Delta t_{1}
$$

This process causes the loss of cooling capacity, which is an adverse heat transfer; the lost cold air flows downward at the temperature $t_{l}$ and speed $v_{l}$, and contacts the cabinet wall to perform flat convection heat exchange. At this time, the cold airflow loses the cooling capacity to cool the cabinet, which is the effective cooling capacity expected during the heat dissipation process. The temperature of the cold air flow continues to rise, resulting in:

$$
t_{2}=t_{0}+\Delta t_{1}+\Delta t_{2}
$$

Cold air flows through the cabinet, and on the other side is affected by the overall ambient gas flow and hot airflow. It is assumed that the cold air flows through the wall surface of the cabinet at a uniform speed; on the one hand, the heat exchange with the wall surface generates effective cooling, on the other hand, it mixes with the hot airflow. Heat exchange causes loss of cooling capacity and changes in hot air temperature. At the end of this process, the temperature of the mixed gas stream is:

$$
t_{3}=t_{0}+\Delta t_{1}+\Delta t_{2}+\Delta t_{3}
$$

Finally, the mixed airflow reaches the upper part of the cabinet and exchanges heat with the cold airflow newly entering the data center. Some of the heat is taken away, and the temperature is slightly reduced to:

$$
t_{4}=t_{3}-\Delta t_{1}=t_{0}+\Delta t_{2}+\Delta t_{3}
$$

Subsequently, the mixed airflow was subjected to complex heat exchange with the environment and was discharged. The temperature change there did not affect the heat dissipation of the cabinet and was not analyzed.

\subsection{Velocity model of cold airflow}

Bringing the known initial velocity into the equation5:

$$
\left\{\begin{array}{c}
v=v_{0} \\
v_{1}=e^{-f\left(H_{1}-H_{2}\right)+\ln v_{0}} \\
v_{2}=e^{-f H_{1}+\ln v_{0}}
\end{array}\right.
$$

$f$ _ Speed factor

$H_{1}$ _Ceiling height, $m$

$H_{2}$ _Cabinet height, $m$

The speed relationship is obtained. According to the altitude and initial speed, the time change relationship of the first three processes can be obtained as follows:

$$
\left\{\begin{array}{c}
\tau_{1}=\frac{v_{0}-v_{1}}{f v_{1} v_{2}} \\
\tau_{2}=\frac{v_{0}-v_{1}}{f v_{0} v_{2}}-\frac{v_{1}-v_{0}}{f v_{1} v_{0}}=\frac{v_{0}-v_{1}}{f v_{0} v_{2}}-\tau_{1} \\
\tau_{3}=2 H_{2}
\end{array}\right.
$$

$\tau_{1}, \tau_{2}, \tau_{3}$ _Time of cold airflow in each process, $s$

The above formula can get the relationship between speed and height, time and height, and then can calculate the relationship between heat transfer and height.

\subsection{Effective cooling efficiency model}

The effective cooling capacity is the two convective heat transfers to the cabinet, which occur in the second and 
third processes. The temperature difference change needs to be obtained as the solution parameter, as shown below:

$$
\Delta t_{1}=\frac{2 a\left(1-e^{-k \tau_{1}}\right)}{1+e^{-k \tau_{1}}}
$$

$a$-Acceleration, $m / s^{2}$

$k$-Temperature change scale factor, 0.077

The temperature difference change of the available heat exchange process is:

$$
\begin{aligned}
& \Delta t=\Delta t_{3}+\Delta t_{2}=\left(t_{2}-t_{n}\right) e^{-k \tau_{3}}+t_{n}-\left(t_{0}-t_{m}\right) e^{-k \tau_{1}}-t_{m} \\
& =\left[\left(t_{0}-t_{m}\right) e^{-k \tau_{1}}+t_{m}-t_{n}\right] e^{-k\left(\tau_{2}+\tau_{3}\right)}+t_{n}-\left(t_{0}-t_{m}\right) e^{-k \tau_{1}}-t_{m}
\end{aligned}
$$

Define the average temperature of the mixed airflow as:

$$
t_{m}=t_{0}+\Delta t_{2}+\Delta t_{3}+\frac{\Delta t_{1}}{2}=t_{0}+\frac{2 \Delta t}{1+e^{-k \tau_{1}}}
$$

Find the effective temperature difference $\Delta \mathrm{t}$ change as:

$$
\Delta t=\frac{\left(t_{n}-t_{0}\right)\left(1-e^{-k\left(\tau_{3}+\tau_{2}\right)}\right)}{1+\frac{2\left(1-e^{-k \tau_{1}}\right)}{1+e^{-k \tau_{1}}}\left(1-e^{-k\left(\tau_{2}+\tau_{3}\right)}\right)}
$$

According to the effective temperature difference, the effective heat exchange amount can be calculated, and then the effective heat dissipation efficiency value can be obtained:

$$
Q=c_{p} q_{m} \Delta t
$$

$Q$ _Effective cooling capacity, $k J$

$c_{p}{ }_{\text {_Specific heat capacity of air, }} J /(\mathrm{kg} \cdot \mathrm{K})$

$$
\eta=\frac{Q}{Q_{1}}
$$
kJ

$Q_{l}$ - Air conditioner provides total cooling capacity,

$\eta$ _Effective cooling efficiency

The higher the efficiency, it proves that the same cooling capacity is provided, and the more cooling capacity is used, the more reasonable the data center size is. In the case of a certain amount of heat generated by the CPU, increasing efficiency can reduce energy consumption.

\section{Solving and verifying the dynamic optimization model}

\subsection{Efficiency and PUE solution for each height series}

Taking the height $\mathrm{H}_{2}$ of the cabinet and the typical heat dissipation amount $Q_{2}$ of the cabinet as fixed values, and using the height of the ceiling as a variable, determine the ceiling height parameter $H_{I}$ and its effective heat dissipation efficiency $\eta$. According to reference 4 , it can be known that the cabinet height is a series of standard heights. Through MATLAB's solution, it is obtained that each standard cabinet height has the corresponding optimal ceiling height. At this time, the heat dissipation efficiency in the equipment room is maximized. The optimized parameter table corresponding to all standard cabinet heights is as follows:

Table 1. $H_{1}, \eta$ and PUE for different $H_{2}$

\begin{tabular}{cccccccc}
\hline $\begin{array}{c}\text { Cabinet height } \\
H_{2}(\mathrm{~m})\end{array}$ & 1.0 & 1.2 & 1.4 & 1.6 & 1.8 & 2.0 & 2.2 \\
\hline $\begin{array}{c}\text { Ceiling height } \\
H_{I}(\mathrm{~m})\end{array}$ & 3.282 & 3.335 & 3.388 & 3.422 & 3.479 & 3.536 & 3.59 \\
\hline $\begin{array}{c}\text { Cooling efficiency } \\
\eta_{(\%)}\end{array}$ & 39.67 & 43.61 & 47.14 & 50.28 & 53.03 & 55.41 & 57.44 \\
\hline PUE & 2.0042 & 1.9586 & 1.9243 & 1.8978 & 1.8771 & 1.8609 & 1.8482 \\
\hline
\end{tabular}

According to the analysis in the above table, as the ceiling height is lower than $3.4 \mathrm{~m}$, the maximum heat dissipation efficiency is less than $50 \%$, that is, under the known building layout, the cooling capacity cannot be fully used to cool the server. Therefore, it is not recommended to consider a ceiling height of less than $3.4 \mathrm{~m}$ during data center construction.

As for a data center with a ceiling higher than $3.4 \mathrm{~m}$, when a higher cabinet height is used, a monotonically increasing cooling efficiency can be obtained, but as the height increases, the increase in efficiency is gradually slowed by the space's thermal diffusion and convection, and the blind increase The height of the ceiling for higher heat dissipation will result in reduced economic efficiency. In addition, as shown in Figure 2, the PUE value is controlled between the standard value 2 and the data center C-level energy consumption value 1.6 as well as the intersection between the PUE curve and the heat dissipation efficiency curve is to the right of 3.5 , so it is recommended to take $3.5 \mathrm{~m}$ as the optimal height for the ceiling of the data center. 


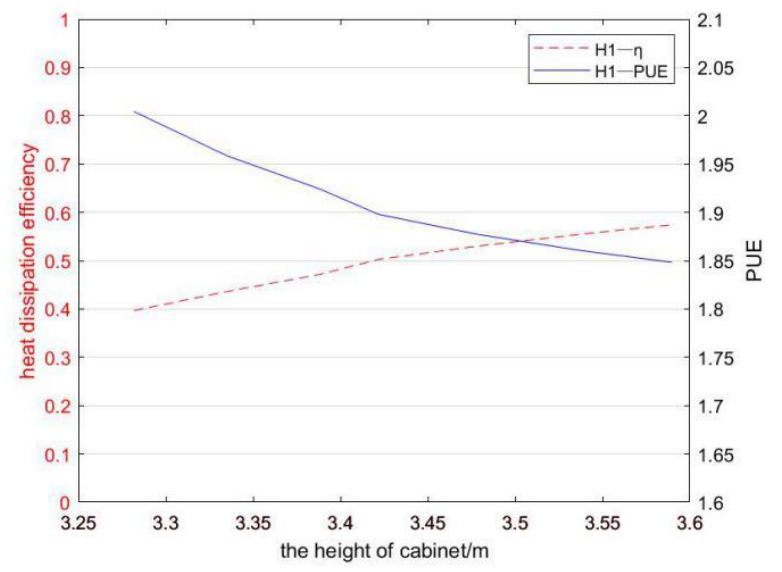

Figure 2. Relationship between heat dissipation efficiency, PUE and ceiling height

\subsection{Verification of optimal ceiling height}

Based on the above analysis, assuming $H_{1}=3.5 \mathrm{~m}$ is unchanged, using the model to prove the optimal cabinet height value, and applying MATLAB plots, we get Figure 3 and 4:

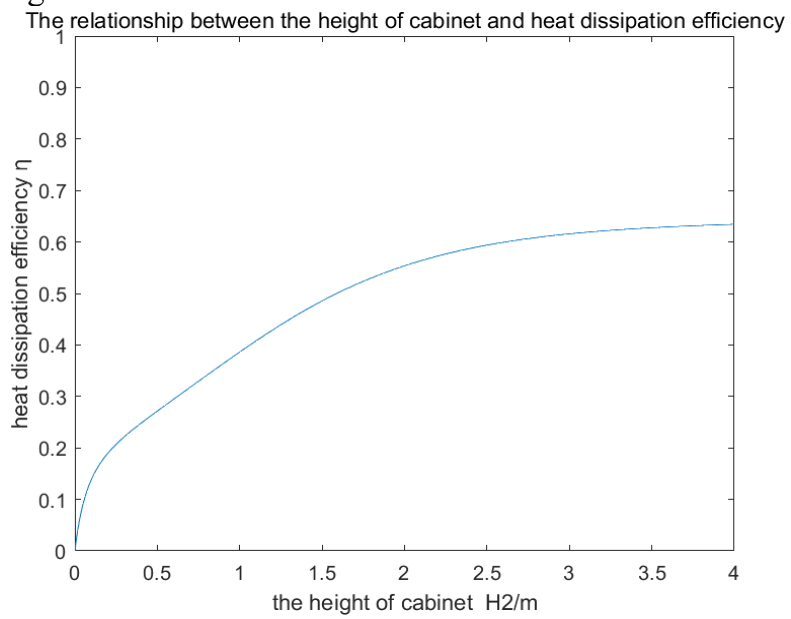

Figure.3 Relation curve between heat dissipation efficiency and cabinet height

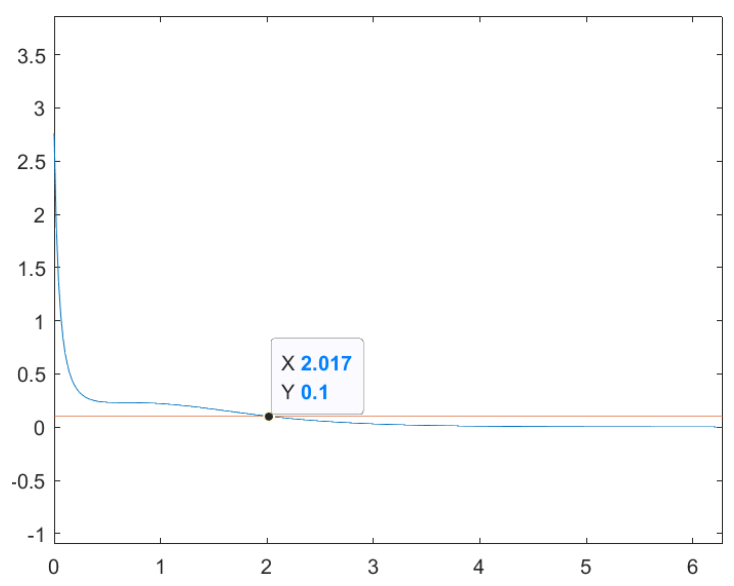

Figure.4 Slope of efficiency curve

From Figure 4, it can be seen that the heat dissipation efficiency increases with the height of the cabinet and increases continuously. When the rate of change of the growth rate is less than $10 \%$, regardless of the effect of increased cabinet height on efficiency, it is obtained that when determining the ceiling height of $3.5 \mathrm{~m}$, the optimal cabinet height should be $2.017 \mathrm{~m}$, and the heat dissipation efficiency is $55 \%$. This result is approximately equal to Table 1, so the optimal design height configuration is: $H_{l}$ $=3.5 \mathrm{~m}, \mathrm{H}_{2}=2.0 \mathrm{~m}$.

\section{Conclusion}

For the improvement of the energy consumption of the refrigeration system during the construction of the green data center, through the solution and verification of the model, the following conclusions are obtained:

1) Different cabinet height series correspond to different ceiling height values. When the ceiling height is in the range of $3.4 \mathrm{~m}-3.6 \mathrm{~m}$, the data center has a relatively high cooling efficiency. At this time, the PUE value is at a high level and meets the green data center standards.

According to the calculation of common standard cabinet heights, the following groups of relatively optimal solutions are obtained:

(a) While the height of the cabinet is $1.6 \mathrm{~m}$, the ceiling height is $3.422 \mathrm{~m}$, which can ensure the maximum efficiency of $50.28 \%$ and PUE of 1.8978 .

(b) While the height of the cabinet is $1.8 \mathrm{~m}$, the ceiling height is $3.479 \mathrm{~m}$, which can ensure that the maximum efficiency is $53.03 \%$, and the PUE is 1.8771 .

(c) While the cabinet height is $2.0 \mathrm{~m}$, the ceiling height is $3.536 \mathrm{~m}$, which can ensure the highest efficiency is $55.41 \%$, and the PUE is 1.8609 .

(d) While the cabinet height is $2.2 \mathrm{~m}$, the ceiling height is $3.59 \mathrm{~m}$, which can ensure the maximum efficiency of $57.44 \%$ and PUE of 1.8482 .

2) The optimal height parameters for the proposed data center construction are $3.5 \mathrm{~m}$ ceiling height and $2 \mathrm{~m}$ cabinet height. At this time, the PUE value is around 1.87 , which is at a relatively high level and has high economic efficiency.

3) For different types of cabinets, the typical heat dissipation capacity is different. The recommended cooling capacity serves as $Q_{1} \geq Q_{2}$ to ensure a moderate amount of cooling capacity, which can meet the expected cooling requirements of this article.

\section{References}

1. The Ministry of Industry and Information Technology, the State Organs Affairs Administration and the National Energy Administration jointly launched the evaluation of the pilot units of the National Green Data Center [J] .Green Packaging, 2017 (08): 15.

2. Yin Ping.Data Center Research (4): Key Performance Indicators, Power Usage Efficiency PUE and EEUE [J] .HVAC, 2017,47 (04): 36-45 + 135.

3. Liu Huan. Design and energy efficiency analysis of 
data center cooling system based on separated heat pipe [D] .University of Chinese Academy of Sciences (Institute of Engineering Thermophysics, Chinese Academy of Sciences), 2017.

4. Li Jin, Cloud Computing Data Center Planning and Design, People's Posts and Telecommunications Publishing House, 2018

5. Chen Xuyong. Simulation analysis of aerodynamics of vacuum maglev train [D] .Southwest Jiaotong University, 2013. 